\title{
Bmpr encodes a type I bone morphogenetic protein receptor that is essential for gastrulation during mouse embryogenesis
}

\author{
Yuji Mishina, ${ }^{1}$ Atsushi Suzuki, ${ }^{2}$ Naoto Ueno, ${ }^{2}$ and Richard R. Behringer ${ }^{1,3}$ \\ ${ }^{1}$ Department of Molecular Genetics, The University of Texas, M.D. Anderson Cancer Center, Houston, Texas 77030 USA; \\ ${ }^{2}$ Faculty of Pharmaceutical Sciences, Hokkaido University, Sapporo 060, Japan
}

Bone morphogenetic proteins (BMPs) are secreted proteins that interact with cell-surface receptors and are believed to play a variety of important roles during vertebrate embryogenesis. Bmpr, also known as $A L K-3$ and $B r k-1$, encodes a type I transforming growth factor- $\beta$ (TGF- $\beta$ ) family receptor for BMP-2 and BMP-4. Bmpr is expressed ubiquitously during early mouse embryogenesis and in most adult mouse tissues. To study the function of Bmpr during mammalian development, we generated Bmpr-mutant mice. After embryonic day 9.5 (E9.5), no homozygous mutants were recovered from heterozygote matings. Homozygous mutants with morphological defects were first detected at E7.0 and were smaller than normal. Morphological and molecular examination demonstrated that no mesoderm had formed in the mutant embryos. The growth characteristics of homozygous mutant blastocysts cultured in vitro were indistinguishable from those of controls; however, embryonic ectoderm (epiblast) cell proliferation was reduced in all homozygous mutants at E6.5 before morphological abnormalities had become prominent. Teratomas arising from E7.0 mutant embryos contained derivatives from all three germ layers but were smaller and gave rise to fewer mesodermal cell types, such as muscle and cartilage, than controls. These results suggest that signaling through this type I BMP-2/4 receptor is not necessary for preimplantation or for initial postimplantation development but may be essential for the inductive events that lead to the formation of mesoderm during gastrulation and later for the differentiation of a subset of mesodermal cell types.

[Key Words: Gastrulation; mesoderm; TGF- $\beta$ gene family; signal transduction]

Received June 5, 1995; revised version accepted October 25, 1995.

Bone morphogenetic proteins (BMPs), decapentaplegic, $60 \mathrm{~A}$, screw, growth and differentiation factors (GDFs), and nodal compose a subgroup within the transforming growth factor- $\beta$ (TGF- $\beta$ ) gene superfamily (Arora et al. 1994; Kingsley 1994a). The BMPs currently consist of eight members (BMP-2 through BMP-8A and BMP-8B). Most of these BMPs are capable of inducing the formation of bone when subcutaneously implanted into rodents (Wozney 1992; Kingsley 1994b). Bone formation occurs through a series of endochondral events initiated by chemotaxis of mesenchymal stem cells into the implantation site (Sampath and Reddi 1981; Reddi 1994). These cells proliferate and differentiate into chondrocytes, whose matrix is calcified and subsequently replaced by the deposition of bone. Thus, the BMP family of proteins possesses potent bone-inducing properties.

BMPs were originally identified by their ability to cause bone differentiation (Urist 1965). Recent studies of

\footnotetext{
${ }^{3}$ Corresponding author.
}

several organisms, however, suggest that several BMPs have other roles during embryogeresis, notably in dorsoventral and/or anterior-posterior axis formation. In Drosophila melanogaster, mutations in decapentaplegic (dpp), which is believed to be a homolog of BMP-2 and BMP-4, cause dorsoventral patterning abnormalities at the blastoderm stage (Ray et al. 1991). The finding that human BMP-4 can rescue the embryonic lethality of $d p p$ null mutants (Padgett et al. 1993) suggests that BMPs may have a comparable role in vertebrate pattern formation. In addition, in Xenopus laevis, BMP-4 can act as a posterior-ventralizing factor in animal cap explant and blastocoele implant assays (Dale et al. 1992; Jones et al. 1992). The expression patterns of BMP-2 and BMP-4 in mouse embryos and of BMP-4 in frog embryos further supports the idea that in vertebrates BMPs have other roles in addition to those in bone formation (Lyons et al. 1989; Jones et al. 1991; Fainsod et al. 1994; Francis et al. 1994; Johansson and Wiles 1995).

Because BMPs are secreted proteins, characterization of their receptors and signal transduction pathways is an 
important step in understanding the role of these proteins in development. Recently, type I and type II receptors for TGF- $\beta$ family ligands have been cloned and shown to have conserved Ser/Thr kinase domains (Mathews and Vale 1991; Attisano et al. 1992, 1993; Lin et al. 1992; Ebner et al. 1993; Franzén et al. 1993; ten Dijke et al. 1993; di Clemente et al. 1994; Suzuki et al. 1994a). In Caenorhabditis elegans, Daf-4 encodes a type II receptor that can bind both human BMP-2 and BMP-4 (Estevez et al. 1993). Mutations in Daf-4 inhibit dauer larva formation (Golden and Riddle 1984). In Drosophila, saxophone $(s a x)$, thick veins $(t k v)$, and punt have recently been cloned and shown to encode type I (sax and $t k v$ ) or type II (punt) TGF- $\beta$ family receptors. It is believed that these proteins are functional dpp receptors because their mutant phenotypes are similar to those of $d p p$ mutants (Ray et al. 1991). Moreover, in COS cells, tkv can bind dpp protein and punt can bind BMP-2 in the presence of either tkv or sax (Brummel et al. 1994; Nellen et al. 1994; Okano et al. 1994; Penton et al. 1994; Xie et al. 1994; Letsou et al. 1995; Ruberte et al. 1995l. These findings suggest that BMP signaling is critical for invertebrate embryonic development, indicating that BMP signaling may also be important during vertebrate embryogenesis.

Recently, a cDNA (TFR-11) was isolated from mouse MC3T3-E1 cells by reverse transcription-polymerase chain reaction (RT-PCR) with degenerate oligonucleotide primers located in the conserved regions of the Ser/ Thr kinase domain of the TGF- $\beta$ and activin receptors (Suzuki et al. 1994b). The same gene was also isolated as Brk-1 (Koenig et al. 1994). The human homolog is known as $A L K-3$ (ten Dijke et al. 1993). TFR-11 encodes a type I transmembrane Ser/Thr kinase receptor that when expressed in COS cells binds both BMP-2 and BMP-4 but not TGF- $\beta$ or activin A (Suzuki et al. 1994b). The gene for this BMP-2/4 receptor (Bmpr) is encoded by 11 exons that span $\sim 38 \mathrm{~kb}$ and maps to mouse chromosome 14 (Mishina et al. 1995). Interestingly, a mutant form of this type I BMP-2/4 receptor acts in a dominant-negative manner to induce the formation of a secondary axis in Xenopus embryos, suggesting that inhibition of BMP signaling by this mutant receptor can alter the fate of embryonic cells toward a more dorsal identity (Maéno et al. 1994; Suzuki et al. 1994b).

To understand the role of Bmpr during vertebrate development, we deleted the first two exons of Bmpr by gene targeting in mouse embryonic stem (ES) cells. Bmpr-mutant embryos developed normally during preimplantation and initial postimplantation stages. At E7.0, however, the homozygous mutants were smaller than normal, and no mesoderm had formed, which ultimately led to embryo death. Cell-cycle analysis revealed that mutant epiblast cells were compromised with respect to cell proliferation. Analysis of tissues derived from Bmpr mutant embryos that had been transplanted into testes and grown in vivo showed that they could differentiate into cell types from all three germ layers. The sizes of the tumors and number of differentiated cell types present, notably those derived from mesoderm, however, were smaller than those of controls. These results suggest that signals transduced by this type I BMP. $2 / 4$ receptor are not necessary up to the initial stages of postimplantation development but may be important for the inductive processes that result in the formation of mesoderm and subsequent differentiation of a subset of mesodermal cell types.

\section{Results}

\section{Bmpr expression pattern}

$B m p r$ is expressed in most adult mouse tissues including bone (ten Dijke et al. 1993; Koenig et al. 1994; Mishina et al., in prep.). Bmpr expression is also detected by Northern blot analysis as early as embryonic day 8.5 (E8.5) (Y. Mishina, J. Rivera-Perez, A. Suzuki, N. Veno, and $\mathrm{R}$. Behringer, in prep.). To determine the spatial expression pattern of $B m p r$, RNA in situ hybridization on whole and sectioned embryos was performed with a probe encoding the extracellular region of Bmpr. At E7.0, $B m p r$ transcripts were detected homogeneously throughout the epiblast and mesoderm (Fig. 1A,B). At E7.5, Bmpr appeared to be expressed ubiquitously, with stronger signals detected in the epiblast, extraembryonic ectoderm, and extraembryonic mesoderm, that is, allantois and

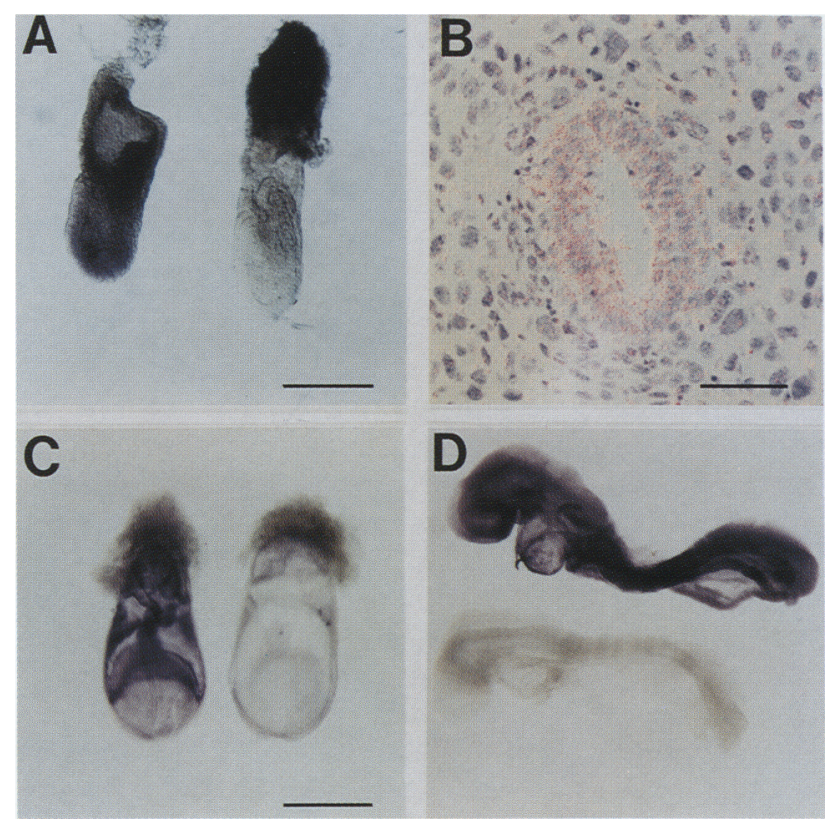

Figure 1. Bmpr expression in early mouse embryos. $(A, B)$ E7.0 embryos $[\mathrm{E} 6.5+11 \mathrm{hr}$, late streak stage. $(A)$ Whole mount; $(B)$ transverse section $]_{;}(C)$ E7.5 embryos $(E 7.5+0 \mathrm{hr}$, late neural plate stage, dorsal view); (D) E8.5 embryos (lateral view) hybridized with a probe that corresponded to the extracellular region of Bmpr. The sense controls are shown in $A, C$ (right), and $D$ (bottom). No signals were detected with a sense-strand probe on the adiacent section for $B$ (data not shown). Bmpr transcripts were distributed ubiquitously between E7.0 and E8.5. Bar, 240 $\mu \mathrm{m}$ in $A ; 60 \mu \mathrm{m}$ in $B$; and $480 \mu \mathrm{m}$ in $C$ and $D$. 
proamniotic canal (Fig. 1C). At E8.5, Bmpr was ubiquitously expressed in all three germ layers along the entire body axis (Fig. 1D). Thus, the expression pattern of $B m p r$ suggests that all regions of the developing mouse embryo may be responsive to BMP signals emanating from maternal and/or zygotic sources.

\section{Generation of a Bmpr mutant allele in the mouse germ line}

To mutate the Bmpr gene in mouse ES cells, we generated a targeting vector that deletes $6.3 \mathrm{~kb}$ of $B m p r$, including the first two exons, by replacing them with a neomycin-resistance expression cassette (Fig. 2A). When the vector is homologously recombined with the endogenous gene, novel EcoRV and BglI sites are introduced (Fig. 2A). Correctly targeted clones can therefore be detected by the presence of an additional $6.3-\mathrm{kb}$ mutant fragment when digested with EcoRV and hybridized with a $3^{\prime}$ probe external to the region of vector homology or by the presence of a $4.6-\mathrm{kb}$ mutant band when digested with $B g l$ and hybridized with a $5^{\prime}$ probe internal to the region of vector homology (Fig. 2B). Correct targeting deletes the first two exons of $B m p r$, which encode the translation initiation start codon, the signal peptide, and $\sim 30 \%$ of the extracellular domain of the receptor. It was predicted that this mutation would functionally inactivate Bmpr. Five correctly targeted ES clones success-
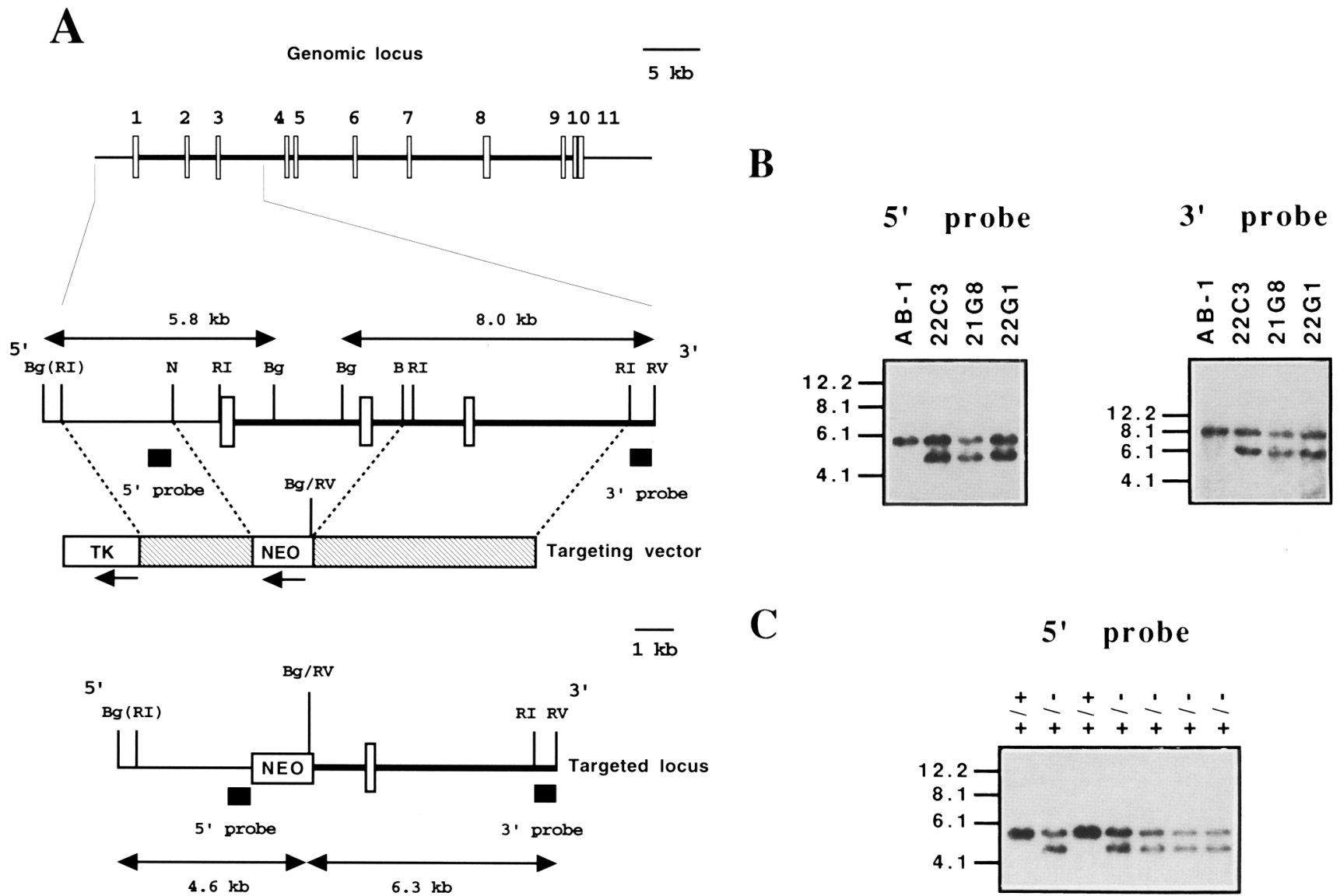

Figure 2. Mutation of the Bmpr locus. $(A)$ Strategy for targeted mutation of the Bmpr locus. (Top) Structure of the Bmpr locus (Mishina et al. 1995). (Open rectangles) Bmpr exons; (thick line) introns. (Middle) Detail of the Bmpr locus showing exons 1-3 and the gene targeting vector. (Hatched regions) Bmpr homologous regions used in the targeting vector; (NEO) PGK-neo expression cassette that introduces novel BglI and EcoRV restriction sites; (TK) MCl-tk expression cassette for negative selection. The arrows beneath NEO and TK indicate the direction of transcription of each cassette. The unique probes for Southern blotting are shown. The sizes of the restriction fragments detected by these probes in wild-type DNA are shown above the locus. (Bottom) Structure of the targeted locus. The sizes of the restriction fragments detected by the Southern blot probes in the targeted locus are shown. (B) Bam $\mathrm{HI}$; $(\mathrm{Bg}) \mathrm{Bg} / \mathrm{I}$; (N) NheI; (RI) EcoRI; (RV) EcoRV. The most 5' EcoRI site (indicated in parentheses) is derived from the multiple cloning region of the $\lambda$ DASH II phage vector. $(B)$ Southern analysis of genomic DNA isolated from ES cell lines and mice derived from heterozygote matings. Correct targeting events are shown for three different ES clones $(22 \mathrm{C} 3,21 \mathrm{G} 8$, and 22G1). (Left) BgII-digested ES cell DNA hybridized with the $5^{\prime}$ probe. The 5.8-kb wild-type and 4.6-kb mutant bands are shown. (Right) BglI/EcoRV-digested ES cell DNA hybridized with the $3^{\prime}$ probe. The $8.0-\mathrm{kb}$ wild-type and $6.3-\mathrm{kb}$ mutant bands are shown. (AB-1) Wild-type ES cell line. (C) Southern blot showing genotypes of newborn mice from a heterozygote mating. BglI-digested tail DNA hybridized with the $5^{\prime}$ probe. The 5.8-kb wild-type and 4.6-kb mutant bands are shown. $1+1+\mid$ Wild-type; $1+1-1$ heterozygous mutant. 
fully contributed to the germ lines of chimeric mice generated by blastocyst injection. The phenotypes of the $B m p r$-mutant mice from three of these independently derived ES clones were identical. The phenotype of the Bmpr mutation was examined on a C57BL/6 $\times 129 / \mathrm{SvEv}$ mixed genetic background, a 129/SvEv inbred genetic background, a genetic background that was $\sim 90 \%$ $\mathrm{C} 57 \mathrm{BL} / 6$, and a $129 / \mathrm{SvEv} \times$ Swiss mixed genetic background and was essentially the same on each of these four different genetic backgrounds.

\section{Early recessive embryonic lethality in $\mathrm{Bmpr}$ mutant mice}

Mice heterozygous for the Bmpr mutation appeared nor$\mathrm{mal}$ and were fertile. In addition, no abnormalities were detected in the skeletons of heterozygous neonates prcpared by alkaline digestion and alizarin red/alcian blue staining for bone and cartilage (data not shown). There were no homozygous mutants among the progeny born from matings between heterozygotes, indicating that Bmpr mutant mice died during embryogenesis (Fig. 2C; Table 1). To characterize this embryonic lethality, timed matings were established and the resulting embryos were dissected free of maternal tissues and genotyped. Abnormal or degenerating embryos were recovered between E7.0 and E8.5 |Fig. 3). The seventh day of gestation is the stage at which gastrulation initiates (Snow 1977). At this stage, the morphologically normal embryos had developed a primitive streak, the region of the embryo where epiblast cells are emigrating between the embryonic ectoderm and the primitive endoderm to form mesoderm. About one-quarter of the embryos at this stage, however, were morphologically abnormal, with no primitive streak, and approximately half the size of the normal embryos (Fig. 3A). In addition, the boundary between the embryonic and extraembryonic portions of the embryos was indistinct in the abnormal embryos. At E8.0, advanced normal embryos had already formed head folds, whereas no organized structures were observed in the abnormal embryos, and they were much smaller and had generated empty sacs composed of parietal endoderm (Fig. 3B). Genotype analysis by PCR revealed that all of the morphologically normal embryos that were recovered from the heterozygote matings between E7.0 and E8.5 were either wild type or heterozygous and all of the morphologically abnormal embryos were homozy-

Table 1. Genotypes of offspring from Bmpr heterozygote matings

\begin{tabular}{lrcr}
\hline & \multicolumn{3}{c}{ Number $(\%)$} \\
\cline { 2 - 4 } ES clone & $+1+$ & $+1-$ & $-1-$ \\
\hline $22 \mathrm{C} 3$ & $40(37)$ & $69 \mid 63)$ & $0|0|$ \\
$21 \mathrm{G} 8$ & $5(33)$ & $10 \mid 67)$ & $0|0|$ \\
$22 \mathrm{G} 1$ & $2(18)$ & $9 \mid 82)$ & $0\lfloor 0)$ \\
Total & $47(35)$ & $88(65)$ & $0|0|$ \\
\hline
\end{tabular}
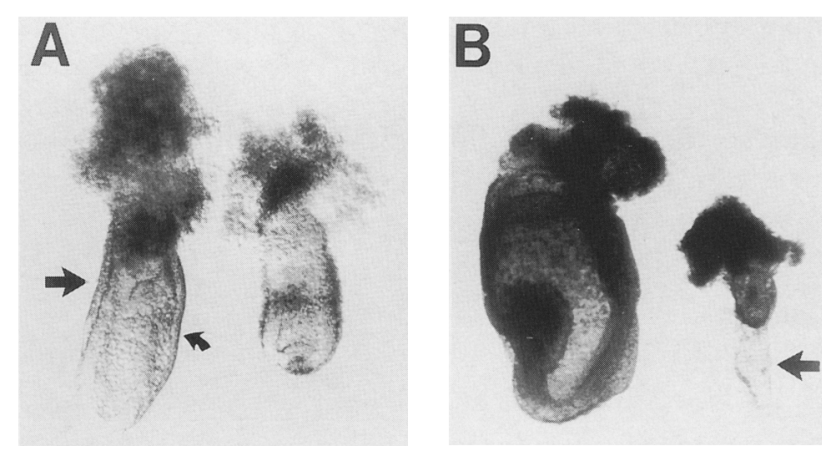

Figure 3. Morphology of $B m p r$ mutant embryos. Whole-mount preparations at E7.0, late streak $|A|$ and E8.0, late head-fold $|B|$ of control (left), and Bmpr mutant (right) embryos. At E7.0, the mutants were smaller than normal and no primitive streak was detected (curved arrow). In addition, the boundary between the embryonic and extraembryonic regions of the control embryo is distinct |straight arrow/, in the wild type but not in the mutant. At E8.0, the mutant embryos were much smaller than normal. Note the sac of parietal endoderm (arrow in $B$ ). Magnification, $50 \times$ in $A$ and $25 \times$ in $B$.

gous for the Bmpr mutant allele (Table 2). Furthermore, no homozygous mutants were recovered at or after E9.5, and an excess of resorption sites were found /data not shown]. These results demonstrate that homozygosity for this Bmpr mutation results in early embryonic lethality and indicate that $B m p r$ is important for postimplantion development around the time of gastrulation.

\section{No mesoderm formation in Bmpr mutant embryos}

The structures of the mutant embryos were characterized in more detail by histological analysis. Twenty-four E5.5 egg cylinder-stage embryos obtained from heterozygote matings were completely serial sectioned, and no structural differences were detected (data not shown). These results suggest that the abnormalities found in the E7.0 homozygous mutant embryos developed between E5.5 and E7.0. Therefore, we focused our attention on E7.0 embryos. At E7.0, control embryos had a primitive streak with mesoderm present between the embryonic ectoderm and visceral endoderm (Fig. 4A,B). Although the size of the yolk sac cavity of mutants was almost the

Table 2. Genotypes of embryos from Bmpr heterozygote matings

\begin{tabular}{|c|c|c|c|c|c|c|}
\hline \multirow[b]{3}{*}{ Stage } & \multicolumn{6}{|c|}{ Number $|\%|$} \\
\hline & \multicolumn{3}{|c|}{ normal embryos } & \multicolumn{3}{|c|}{ abnormal embryos } \\
\hline & $+1+$ & $+1-$ & $-1-$ & $+1+$ & $+1-$ & $-1-$ \\
\hline E7.0 & $5|33|$ & $8 \mid 53)$ & $0\langle 0\}$ & $0(0)$ & $0(0)$ & $2(14)$ \\
\hline E7.5 & $6 \mid 21)$ & $15\lfloor 52 \mid$ & $0|0|$ & $0(0)$ & $0\langle 0\}$ & $8(27)$ \\
\hline $\mathrm{E} 8.5$ & $7 \nmid 18\}$ & $26\lfloor 68 \mid$ & $0|0|$ & $0(0)$ & $0\langle 0\rangle$ & $5(13)$ \\
\hline Total & $18 \mid 22\}$ & $49\lfloor 60\}$ & $0(0)$ & $0(0)$ & $0(0)$ & $15(18)$ \\
\hline
\end{tabular}



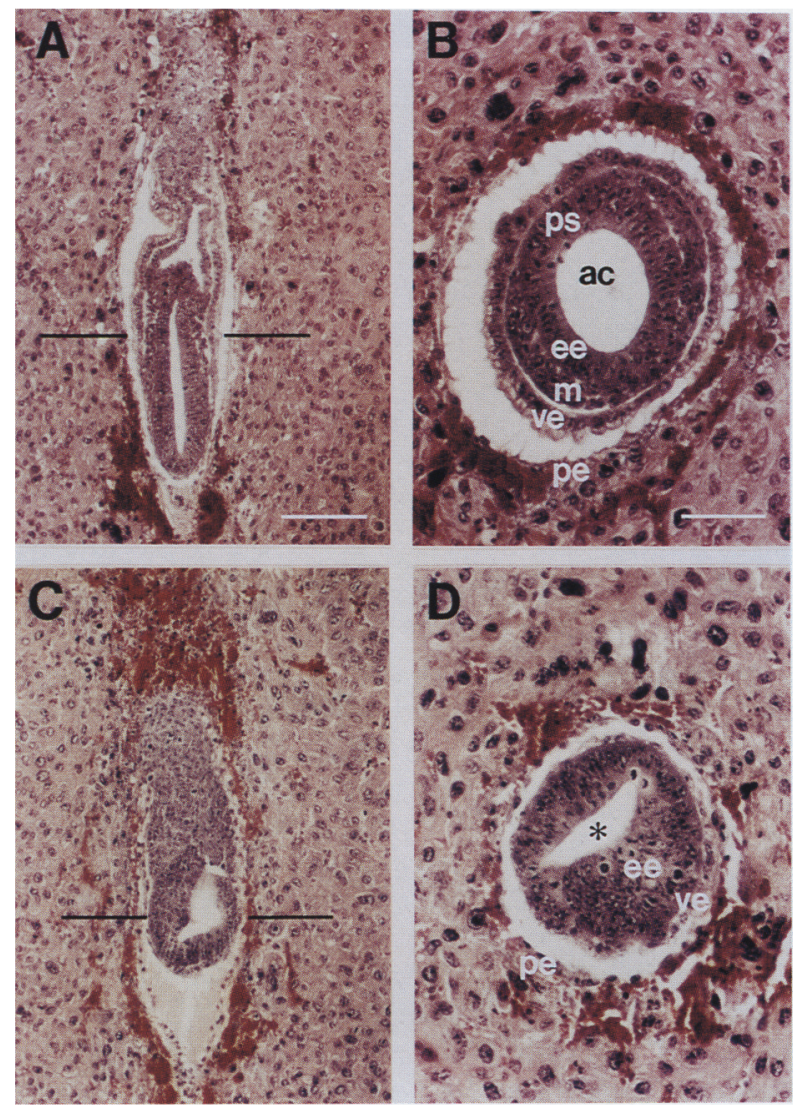

Figure 4. Histological analysis of E7.0 Bmpr mutant embryos Histological comparison of control $\langle A, B|$ and mutant $|C, D\rangle|\mathrm{E}\rangle .0$ embryos in the uterus. $(A, C)$ Sagittal sections; $(B, D)$ transverse sections. The solid lines in the sagittal sections indicate the approximate positions of the transverse sections. $|a c|$ Amniotic cavity; (ee) embryonic ectoderm; $|\mathrm{m}|$ mesoderm; (pe) parictal endoderm; (ps) primitive streak; |vel visceral endoderm; ${ }^{*}$ proamniotic canal. Bar, $120 \mu \mathrm{m}$ in $A$ and $C$ and $60 \mu \mathrm{m}$ in $B$ and $D$.

same as in controls, the size of the embryonic regions were only approximately half as large (Fig. 4A,C). Both parietal and visceral endoderm had formed in the mutants, although the visceral endoderm was thinner |Fig. $4 \mathrm{D}$. Strikingly, no mesoderm was observed in any of the mutant embryos. In the control embryos, the epiblast consisted of a single layer of cells. In the mutant embryos, however, the epiblast was thickened with multiple layers of cells, particularly in the distal portion (Fig. $4 \mathrm{C}, \mathrm{D}$ |. In addition, some of the cells in the thickened region had pyknotic nuclei, suggesting cell death (Fig. 4D). At E7.5, the mutant embryos still had not formed mesoderm and had begun to degenerate (data not shown). Thus, by morphological and histological criteria, no mesoderm formed in the Bmpr-homozygous mutant embryos.

To determine, at the molecular level, if mesoderm had formed in the Bmpr homozygous mutants, we examined the expression of four mesodermal marker genes. Four or five mutant E7.0 embryos per probe were examined by whole mount in situ hybridization for the expression of goosecoid, Brachyury, Lim1, and HNF-3ß, which are expressed in the mesoderm of early-to-late streak-stage embryos (Herrmann 1991; Blum et al. 1992; Sasaki and Hogan 1993; Barnes et al. 1994). None of these four mesoderm markers was expressed in any of the mutant embryos examined, whereas control embryos displayed the characteristic expression patterns for each of the probes (Fig. 5). Therefore, these results further confirmed that no mesoderm had formed in the Bmpr mutant embryos.

\section{In vitro growth of Bmpr mutant preimplantation embryos}

The observations presented above suggested that signaling through this type I BMP-2/4 type I receptor was essential for mesoderm formation. It has been reported that 1400-1500 cells must accumulate in the epiblast to initiate gastrulation (Power and Tam 1993). Therefore, if signaling through this receptor is necessary for cell proliferation, the absence of the signal may result in the production of fewer epiblast cells and therefore indirectly to a failure of mesoderm formation. To address this hypothesis, we examined the growth characteristics of mutant blastocysts cultured in vitro.

Forty-six E3.5 blastocysts from Bmpr heterozygote matings were placed individually in drops of M15 medium on gelatinized plates for in vitro culture. Six days later, the morphology of the embryos was noted and DNA was extracted for genotyping by PCR. All of the

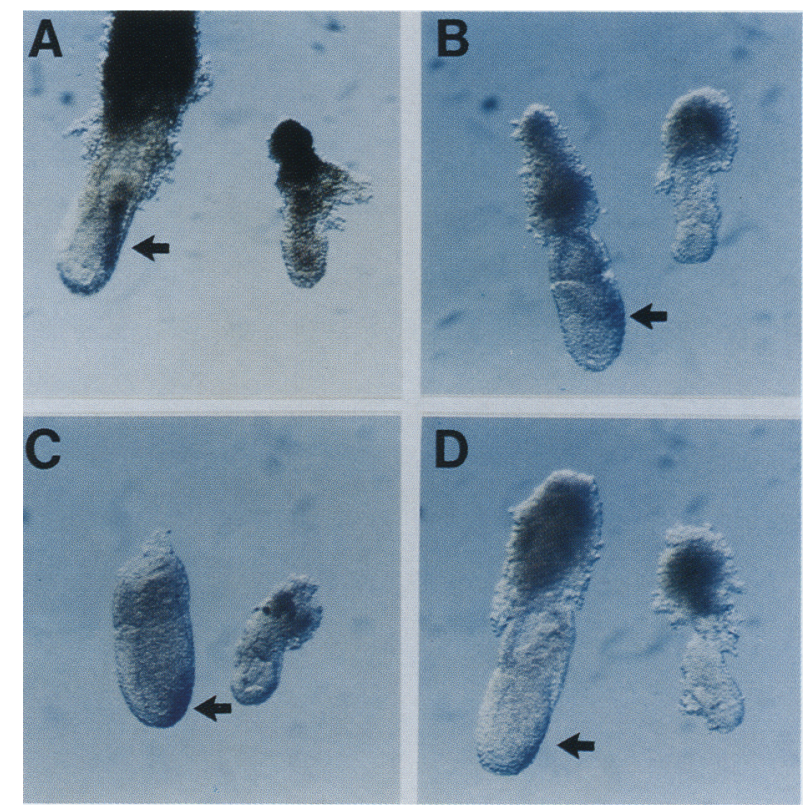

Figure 5. Whole-mount in situ hybridization analysis of mesodermal markers. Expression patterns of Brachyury $(A \mid$, goosecoid $|B|, \operatorname{Lim} 1 \mid C)$, and $H N F-3 \beta(D)$ at E. 7.0 in presumptive Bmpr homozygous mutant embryos (right) compared with normal littermates (left). These four marker genes were expressed in mesodermal tissues of wild-type embryos (arrows). No transcripts for these four marker genes were detected in the Bmpr mutant embryos. Magnification, $50 \times$ 
blastocysts examined formed adherent colonies. Seventeen of these 46 colonies $(37 \%)$ were determined to be homozygous for the Bmpr mutant allele, whereas 5 $(11 \%)$ and $24(52 \%)$ were wild-type and heterozygous, respectively. The shape and size of the mutant colonies were indistinguishable from those of wild-type and heterozygous colonies (data not shown). Under these culture conditions, endoderm differentiation was detected in all three different genotypes (data not shown). These results suggest that the proliferative abilities of homozygous mutant cells derived from E3.5 blastocysts were normal and that these cells were able to proliferate for at least 6 days under these culture conditions.

\section{Altered epiblast cell proliferation in Bmpr mutant gastrulation-stage embryos}

Although the proliferation of cells derived from mutant blastocysts cultured in vitro was indistingushable from controls, the size difference of E7.0 mutant embryos in comparison to controls suggested that cell proliferation in postimplantation mutant embryos was decreased. To address this hypothesis, we examined cell proliferation by the incorporation of 5-bromo- 2 '-deoxyuridine (BrdU) at E6.5 (noon of the sixth day of gestation), the time that gastrulation initiates under the light-cycle conditions of our mouse colony. We chose this stage because morphological abnormalities of mutant embryos are less clear, that is, it is difficult to distinguish mutant embryos from embryos that are simply delayed in development. This should reduce the chances of measuring cell proliferation changes because of secondary events that would occur in a degenerating embryo. Embryos were genotyped by RNA in situ hybridization of sections with the Bmpr probe that was used for the above expression studies. This allows the identification of homozygous mutants from wild-type and heterozygote embryos. As shown in Figure 6, nearly all nuclei of the cells of the epiblast of $30 / 37(81 \%)$ wild-type/heterozygote embryos were labeled with BrdU after a 20 -min or 1 -hr exposure. The remaining $19 \%$ of the wild-type/heterozygote embryos

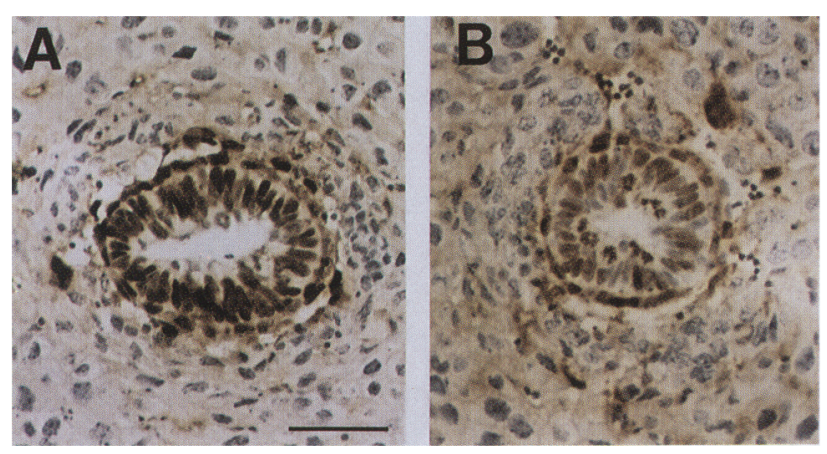

Figure 6. Cell-cycle alterations in Bmpr mutant embryos. Transverse sections of E6.5 wild-type/heterozygote $|A|$ and homozygous mutant $|B\rangle$ embryos visualized for the incorporation of BrdU into nuclei (brown). Unlabeled nuclei appear blue as a result of counterstaining with hematoxylin. Bar, $60 \mu \mathrm{m}$. incorporated label into only about $70 \%-80 \%$ of their nuclei. We presume that these are wild-type/heterozygote embryos that are delayed in their development. In the case of the homozygous mutant embryos, $10 / 10(100 \%)$ showed $70 \%-80 \%$ nuclear labeling (Fig. 6B). Abnormally shaped nuclei were also observed in the abnormal embryos (Fig. 6B). These results indicate that the proliferative ability of $B m p r$ mutant epiblast cells is already decreased by $\mathrm{E} 6.5$ before overt morphological abnormalities become prominent.

\section{Growth and differentiation potential of Bmpr mutant embryos grown ectopically in testes}

When embryos are transplanted at ectopic sites into histocompatible mice, they can develop into teratomas and teratocarcinomas (Tam 1993). This is a useful in vivo assay for examining the growth and cell differentiation potential of mutant embryos (Holdener et al. 1994). Therefore, to determine the ability of the Bmpr mutant embryos to differentiate into various cell types, we transplanted morphologically normal and mutant late E6.5 embryos individually into the testes of adult male mice. After $\sim 8$ weeks, the testes were fixed and processed for histological analysis. DNA extracted from a region of the teratoma was used for genotyping by PCR. The sizes of the tumors derived from the wild-type and heterozygous embryos varied from 0.5 to $3.0 \mathrm{~cm}$ in diameter, whereas the size of the tumors derived from the mutant embryos never exceeded $0.5 \mathrm{~cm}$. Of 18 wild-type and heterozygous embryos, $14(78 \%)$ generated tumors with tissues derived from all three germ layers, for example, neural tissue, skin, muscle, bone, cartilage, gut, and pancreas (Fig. 7; Table 3). In contrast, only eight of eighteen (44\%) mutant embryos produced teratomas and with fewer tissue types (Fig. 7; Table 3). They contained keratinized epithelium (Fig. 7C), hair and hair follicles (Fig. 7D), gut surrounded by smooth muscle (Fig. 7E), and neural tissue (Fig. $7 \mathrm{Cl}$. Teratomas derived from wild-type and heterozygous embryos developed both undifferentiated and differentiated neural tissue, whereas abnormal embryos developed only differentiated neural tissue. Significantly, none of the mutant embryo-derived teratomas formed skeletal or cardiac muscle, a tissue type that was readily produced in the control-derived embryos. In addition, an area containing cartilage was found in only one of the nine mutant embryo-derived teratomas, and this area was significantly smaller than the cartilage structures derived from the control embryos (Fig. 7B,F). These results suggest that cells derived from E6.5 Bmpr mutant embryos are able to proliferate in the testis and can differentiate into not only ectodermally and endodermally derived cell types but also mesodermally derived cell types. The proliferative and differentiative abilities of these mutant cells, however, appear to be limited.

\section{Discussion}

The data presented here show that soon after implantation, Bmpr homozygous mutant embryos failed to pro- 


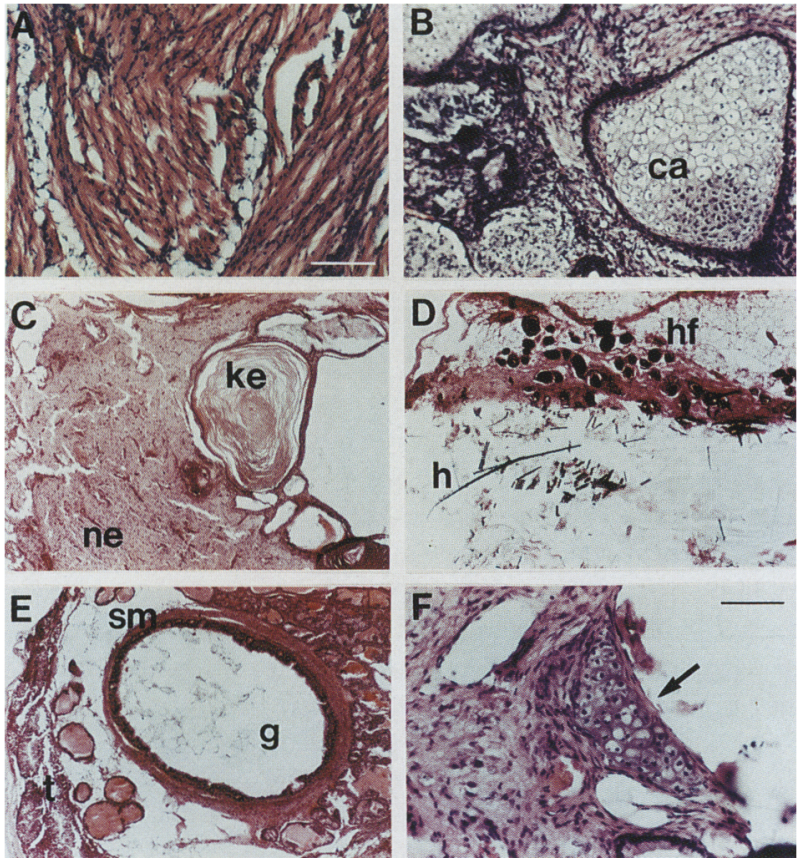

Figure 7. Histological analysis of teratomas derived from E6.5 $B$ mpr mutant embryos. Teratomas derived from late $\mathrm{E} 6.5$ normal embryos $(A, B)$ and mutant embryos $(C-F)$ transplanted into testes and analyzed 8 weeks after the transfer. Muscle tissue arising from a normal embryo is shown in $A$. A small region of cartilage $(F$, arrow $)$ had arisen from a mutant embryo. (ca) Cartilage; (g) gut; (h) hair; (hf) hair folicle; (kel keratinized epithelium; $\mid$ ne $\mid$ neuroectoderm; $(\mathrm{sm})$ smooth muscle; $(\mathrm{t})$ testis tubule. Bar, $120 \mu \mathrm{m}$ in $A$ and $B-E \mid$ and $60 \mu \mathrm{m}$ in $F$.

duce mesoderm, which subsequently led to early embryonic death. The primary lesion caused by this mutation appears to be in the regulation of epiblast-cell proliferation of egg cylinder-stage embryos. In Xenopus, BMP-4 has been shown to act as a ventralizing factor (Dale et al. 1992; Jones et al. 1992), and a mutant form of Bmpr acts in a dominant-negative manner to induce the formation of a hyperdorsalized secondary axis (Graff et al. 1994; Maéno et al. 1994; Suzuki et al. 1994b|. These results suggest that BMP-4 signals can alter the fate of embryonic cells toward a more posterior-ventral identity. We therefore expected Bmpr homozygous mutants to have abnormal patterning of mesodermal tissues, i.e., less posterior and/or ventral mesoderm. In contrast, the results shown here clearly demonstrate that the formation of mesoderm was not induced in the Bmpr mutant embryos at all. These results demonstrate that signaling through this type I BMP receptor is essential for the transition from the symmetric egg-cylinder stage to the asymmetric gastrula stage of mouse embryogenesis.

\section{Roles for BMP-2/4 receptor signaling}

Our studies show that signaling through the BMP-2/4 receptor is not necessary for cell proliferation up to the formation of the egg cylinder; thereafter it becomes essential. In normal embryos at E5.5 (egg cylinder) the av- erage length of the cell-cycle for epiblast cells is $11.5 \mathrm{hr}$, whereas at E6.5 (gastrulation) the average length is significantly shortened to $4.4 \mathrm{hr}$ (Snow 1977). The length of the cell-cycle of epiblast cells in the E6.5 mutant embryos was already increased prior to the stage when mesoderm formation should occur. The decrease in cellcycle length between E5.5 and E6.5 in normal embryos and the increase in cell-cycle length observed in the Bmpr mutants suggests that signaling through this receptor is required for shortening the length of the cell cycle to rapidly increase the number of epiblast cells at E6.5.

The development of abnormalities (i.e., absence of mesoderm formation| between E5.5 and E7.0 suggests that signaling through $\mathrm{Bmpr}$ is essential for the inductive processes that lead to mesoderm formation. One possibility is that Bmpr is part of a signal transduction pathway that is directly required for mesoderm induction. If signaling through this receptor was solely necessary for mesoderm induction, Bmpr mutant embryos might have been predicted to have developed excessive ectoderm, as is the case for nodal and msd mutant embryos (Conlon et al. 1994; Holdener et al. 1994). At E7.0, however, the Bmpr mutants were smaller than control embryos. An alternative possibility is that signaling through this receptor is necessary for epiblast cell proliferation, which may be indirectly essential for mesoderm induction either by generating enough epiblast cells to initiate gastrulation or by altering the phenotype of these cells so that they become competent to respond to mesoderm-inducing signals. In the case of $H N F-4$ mutant mice, the initiation of mesoderm formation is delayed because of cell death in the embryonic ectoderm. These mutant embryos do form mesoderm, but 1 day later than normal, probably because it takes longer for the number of cells in the epiblast to reach the necessary threshold /Chen et al.

Table 3. Tissue types in teratomas derived from Eo. 5 embryos

\begin{tabular}{lcc}
\hline & Normal $^{a^{a}}$ & Abnormal $^{\mathrm{b}}$ \\
\hline Embryos transplanted & 18 & 18 \\
Tumor developed & 14 & 8 \\
Ectodermal & 14 & 8 \\
$\quad$ neuroectoderm & 11 & 4 \\
keratinized epithelium & 6 & 2 \\
hair \& follicles & 1 & 3 \\
retina & 2 & 0 \\
Mesodermal & 11 & 2 \\
bone & 3 & 0 \\
cartilage & 5 & 1 \\
skeletal/cardiac muscle & 9 & 0 \\
smooth muscle & 1 & 1 \\
Endodermal & 3 & 1 \\
rectum & 2 & 0 \\
gut & 1 & 1 \\
pancreas & 1 & 0 \\
\hline
\end{tabular}

${ }^{a}$ Wild-type or heterozygous mutant embryos.

bHomozygous mutant embryos. 
1994). The Bmpr mutants, however, showed no evidence of mesoderm formation even at E8.0. Our data appear to be most consistent with an indirect requirement for $B m p r$ in mesoderm induction because of the epiblast proliferation defect observed prior to the stage of gastrulation.

E6.5 Bmpr mutant embryos could differentiate into some mesodermally derived cell types when grown ectopically in testes, suggesting either that $B m p r$ is not essential for mesoderm formation or that the testicular environment can provide alternative signals that promote the differentiation of mesodermal cell types. Another type I BMP-2/4 receptor CFK-43a /also known as ALK -6$)$ is expressed in a more restricted pattern, may provide a compensatory signaling pathway for mesoderm formation in Bmpr mutant embryos when grown in the testes (ten Dijke et al. 1994; Yamaji et al. 1994; Dewulf et al. 1995). One consistent finding from the testicular transplant experiments was the impaired growth of the mutant embryos in comparison with the normal embryos, suggesting a deficit in cell proliferation potential that is independent of mesoderm formation (i.e., even the mesoderm that forms in the tumors appears to grow slowly, so the proliferation defect is not confined only to mutant epiblast cells|. In addition, the absence of specific mesodermally derived cell types, notably skeletal and cardiac muscle and the very low frequency of cartilage formation, in the teratomas suggests that this receptor may be part of a signaling pathway that is essential for the differentiation of these cell types later in development.

\section{Comparisons of phenotypes of Bmpr mutant and $\mathrm{BMP}-4$ mutant mice}

Recently, BMP-4-mutant mice have been generated (Winnier et al. 1995). BMP-4 mutants die during embryogenesis with a highly variable phenotype possibly depending in part upon their genetic background. Whereas some BMP-4 mutants could develop to the neural fold/ early somite stage, and some had even turned and had a beating heart, the most severe phenotype was characterized as an arrest at the egg-cylinder stage somewhat similar to the phenotype of the Bmpr mutants. A small amount of extraembryonic mesoderm, however, could be distinguished in these BMP-4 mutant embryos (Winnier et al. 1995). The range of phenotypes observed for the BMP-4 mutant mice suggested that BMP-4 signals are essential for different processes at different stages in mouse embryogenesis. Winnier et al. (1995) suggested that the earliest process requiring a BMP-4 signal is the proliferation of epiblast cells. Our finding that Bmpr mutants also have epiblast-cell proliferation defects suggests that BMP-4 and Bmpr are components of the same signaling pathway that is essential in the early mouse embryo.

The range of phenotypes observed in the BMP-4 mutants is in contrast to the very consistent phenotype of the Bmpr mutant mice, even on four different genetic backgrounds. One possible explanation for these differences is that in the absence of BMP-4, another ligand can provide compensatory functions. For example, the absence of BMP-4 signals could be partly compensated by BMP-2 signals because both ligands can bind to the same receptor (Suzuki et al. 1994b; ten Dijke et al. 1994). Therefore, the range of phenotypes of BMP-4 mutant mice could be explained by variations in the expression levels of BMP-2 in different genetic backgrounds. In contrast, the absence of a BMP-2/4 receptor would prevent the rescue by these ligands and lead to a more severe and consistent phenotype. An alternative hypothesis is that the BMP-4 mutant phenotype can be partially rescued by ligands supplied from the mother, as has been reported for TGF- $\beta$ mutant mice (Shull et al. 1992; Letterio et al. 1994|. This is an intriguing possibility because BMP-4 is known to be expressed in decidual cells during gastrulation (Jones et al. 1991). The altered incorporation of BrdU in the epiblast of the Bmpr mutant embryos was not localized in any particular region, suggesting that a homogenously distributed ligand perhaps maternal decidually derived BMP-4 was essential for epiblast proliferation. Interestingly, zygotic expression of BMP-4 is localized to the posterior primitive streak at E6.5 /Winnier et al. 19951. This focal source of BMP-4 may subsequently interact with a subset of Bmpr-expressing cells to initiate the primitive streak (mesoderm formation) and/or maintain this structure.

In summary, the results from this study begin to characterize the TGF- $\beta$ family signaling pathways that are active in the early mouse embryo and provide the first example of a TGF- $\beta$ superfamily receptor that is essential for viability during early vertebrate embryogenesis (Matzuk et al. 1995). Finally, the widespread expression of $B m p r$ and the variety of roles that can now be assigned to BMPs (Kingsley et al. 1992; Winnier et al. 1995) suggests that Bmpr may be useful for in vivo studies of BMP function using tissue-specific loss-of-function strategies.

\section{Materials and methods}

Mutagenesis of the Bmpr gene in mouse ES cells

A $129 /$ SvEv mouse genomic library was screened with the fulllength TFR-11 cDNA (Suzuki et al. 1994b). Eight positive $\lambda$ clones were isolated, and the genomic organization of Bmpr was characterized (Mishina et al. 1995). A 3.0-kb EcoRI-NheI 5' fragment and a 6.0-kb BamHI-EcoRI $3^{\prime}$ fragment were used to construct a replacement gene targeting vector (Fig. 1A). A PGKneobpA resistance expression cassette was inserted in reverse orientation relative to the direction of Bmpr transcription between the two Bmpr regions (Soriano et al. 1991). An MC1tkpA herpes simplex virus thymidine kinase expression cassette was added onto the short arm of homology to enrich for homologous recombinants by negative selection with 1-(2-deoxy-2-fluoro- $\beta$ D-arabinofuranosyl)-S-iodouracil (FIAU) (Mansour et al. 1988). The targeting vector can be linearized at a unique NotI site outside of the homology. Twenty-five micrograms of linearized targeting vector was electroporated into $10^{7} \mathrm{AB}-1 \mathrm{ES}$ cells that were subsequently cultured in the presence of G418 and FIAU on mitotically inactivated STO fibroblasts (McMahon and 
Bradley 1990; Soriano et al. 1991|. Four hundred G418/FIAU resistant ES clones were initially screened by $E c o R V-B g l l$ digestion and hybridized with a unique 3' probe external to the region of vector homology. Correctly targeted clones were then expanded for further Southern blot analysis by BglI digestion and hybridization with a unique $5^{\prime}$ probe internal to the vector homology. Thirteen correctly targeted ES clones were identified.

\section{Generation of chimeric mice and germ-line} transmission of the Bmpr mutant allele

Seven of the Bmpr mutant ES clones were microinjected into C57BL/6J blastocysts, and the resulting chimeric embryos were transferred to the uterine horns of day 2.5 pseudopregnant foster mothers (Bradley 1987). Chimeras were identified among the resulting progeny by their agouti fur (ES derived) and were subsequently bred with $\mathrm{C} 57 \mathrm{BL} / 6$ mates. Five of the mutant $\mathrm{ES}$ clones $(22 \mathrm{~F} 10,22 \mathrm{C} 3,21 \mathrm{G} 8,22 \mathrm{Gl}$, and $22 \mathrm{D} 1)$ were found to be capable of contributing to the germ lines of chimeric mice. Tail DNA from the agouti pups that resulted from these matings was analyzed by Southern blot with either of the probes used to identify Bmpr heterozygotes. E9.5 and older embryos from heterozygote matings were genotyped by Southern blotting of yolk sac DNA. Earlier stage embryos were genotyped by PCR amplification of yolk sac DNA with the Bmpr-specific primers 5'-AAGGGCTCAGGAGAATGGG-3' and 5'-TGCTGGATTGCTGGTTGGAG-3' and the neo primers $5^{\prime}$-TGAATGAACTGCAGGACGAGG-3' and 5'-AAGGTGAGATGACAGGAGATCC-3'. Chimeras were also bred with $129 /$ SvEv females to establish the Bmpr mutation on a $129 / \mathrm{SvEv}$ inbred genetic background. Mice carrying the Bmpr mutation were also backcrossed to $\mathrm{C} 57 \mathrm{BL} / 6$ mice to generate a congenic mouse line. Generation $4 \mathrm{Bmpr}$ heterozygotes at an inbreeding coefficient of 0.938 , were used in this study. The chimeras were also bred with Swiss females (Taconic Farms, Germantown, NY) to establish the Bmpr mutation on a Swiss outbred genetic background. The Swiss outbred animals carrying the Bmpr mutation used in this study had been backcrossed to Swiss mice three times.

\section{Whole-mount and sectioned in situ hybridization}

Whole-mount in situ hybridization was performed by the protocol described by Conlon and Rossant (1992). The following digoxygenin-labeled probes were used for the whole-mount in situ hybridization studies: Bmpr (Suzuki et al. 1994b), Brachyury (Herrmann 1991), goosecoid (Shawlot and Behringer 1995), Lim 1 (Barnes et al. 1994), and HNF3 (Sasaki et al. 1993). For $B m p r$, the extracellular region was used as a probe to avoid cross hybridization with other members of Ser/Thr kinase family. In situ hybridization on sectioned material was also performed by the protocol described by Zhao et al. \{1993|. The same $B m p r$ probe used for whole-mount studies was labeled with $\left[{ }^{35}\right.$ S]-UTP. Slides were developed after a 10-day exposure and counterstained stained with hematoxylin.

\section{Histological analysis}

Embryos were processed for histological analysis as described by Kaufman (1990). Briefly, the embryos were fixed in Bouin's fixative, dehydrated, and embedded in paraffin. Transverse sections $(7-\mu \mathrm{m}$ thick) and sagittal sections $(5-\mu \mathrm{m}$ thick $)$ were cut and stained with hematoxylin and eosin.

\section{BrdU labeling of embryos}

BrdU labeling of cells in the $S$ phase of the cell cycle was performed according to the protocol described by Hayashi et al. (1988). BrdU (100 $\mu \mathrm{g} / \mathrm{gram}$ of body weight) was injected intraperitoneally into pregnant females at E6.5. The females were sacrificed $20 \mathrm{~min}$ or $\mathrm{l} \mathrm{hr}$ after injection, and the embryos were removed, fixed with $4 \%$ paraformaldehyde, embedded in paraffin, and sectioned at $7 \mu \mathrm{m}$. The sections were reacted with anti-BrdU antibody (Becton-Dickinson). Binding of the antibody was visualized with a biotinylated antibody against mouse IgG and avidin conjugated peroxidase (Vectastain). After the peroxidase reaction with $3,3^{\prime}$-diaminobenzidine, sections were counterstained with hematoxylin and the ratio of labeled to total nuclei per section was quantitated. The genotype of each embryo was determined by RNA in situ hybridization of adjacent sections as mentioned above.

\section{Embryo transplants into testes}

E6.5 embryos were isolated from matings between Bmpr heterozygotes and classified as morphologically normal (presum. ably wild-type or heterozygous) or abnormal (presumably mutant . One normal embryo was transplanted into the right testis and one abnormal embryo was transplanted into the left testis of a $B 6 \times 129 F_{1}$ adult male. Seven to eight weeks later, the testes were removed, fixed in $10 \%$ buffered formalin, and embedded in paraffin for histological analysis as described above. A small portion of the teratoma was scratched from the paraffin section, and DNA was extracted and then genotyped by PCR.

\section{Acknowledgments}

We thank Allan Bradley for the AB-1 ES and SNL 76/7 STO cell lines; Bernhard Herrmann, Brigid Hogan, Hiroshi Sasaki, Jaime Rivera-Pérez, and William Shawlot for the in situ hybridization probes; Hongbing Zhang and Allan Bradley for sharing unpublished information; Brigid Hogan for helpful comments on the manuscript; Mark McArthur and Milton Finegold for advice on the teratoma histology; Qi Zhao for advice on in situ hybridization of sectioned material; and Yoshiko and Kanade Mishina for encouragement. This work was supported by grants from the National Institutes of Health (HD30284) and the Sid W. Richardson Foundation to R.R.B.

The publication costs of this article were defrayed in part by payment of page charges. This article must therefore be hereby marked "advertisement" in accordance with 18 USC section 1734 solely to indicate this fact.

\section{References}

Arora, K., M.S. Levine, and M.B. O'Connor. 1995. The screw gene encodes a ubiquitously expressed member of the TGF- $\beta$ family required for specification of dorsal cell fates in the Drosophila embryo. Genes \& Dev. 8: 2588-2601.

Attisano, L., J.L. Wrana, S. Cheifetz, and J. Massagué. 1992. Novel activin receptors: Distinct genes and alternative mRNA splicing generate a repertoire of serine/threonine kinase receptors. Cell 68: 97-108.

Attisano, L., J. Cárcamo, F. Ventura, F.M.B. Weis, J. Massagué, and J.L. Wrana. 1993. Identification of human activin and TGF $\beta$ type I receptors that form heterodimeric kinase complexes with type II receptors. Cell 75: 671-680.

Barnes, J.D., J.L. Crosby, C.M. Jones, C.V.E. Wright, and B.L.M. Hogan. 1994. Embryonic expression of Lim-1, the mouse homolog of Xenopus Xlim-1, suggests a role in lateral meso- 
derm differentiation and neurogenesis. Dev. Biol. 161: 168178.

Blum, M., S.J. Gaunt, K.W.Y. Cho, H. Steinbesser, B. Blumberg, D. Bittner, and E.M. De Robertis. 1992. Gastrulation in the mouse: The role of the homeobox gene goosecoid. Cell 69: 1097-1106.

Bradley, A. 1987. Production and analysis of chimeric mice. In Teratocarcinomas and embryonic stem cells: A practical approach (ed. E.J. Robertson), pp. 113-151. IRL Press, Oxford, UK.

Brummel, T.J., V. Twombly, G. Marqués, J.L. Wrana, S.J. Newfeld, L. Attisano, J. Massagué, M.B. O'Connor, and W.M. Gelbart. 1994. Characterization and relationship of dpp receptors encoded by the saxophone and thick veins genes in Drosophila. Cell 78: 251-261.

Chen W.S., K. Manova, D.C. Weinstein, S.A. Duncan, A. S. Plump, V.R. Prezioso, R. F. Bachvarova, and J.E. Darnell Jr. 1994. Disruption of the HNF-4 gene, expressed in visceral endoderm, leads to cell death in embryonic ectoderm and impaired gastrulation of mouse embryos. Genes \& Dev. 8: 2466-2477.

Conlon, R.A. and J. Rossant. 1992. Exogenous retinoic acid rapidly induces anterior ectopic expression of murine Hox-2 genes in vivo. Development 116: 357-358.

Conlon, F.L., K.M. Lyons, N. Takaesu, K.S. Barth, A. Kispert, B. Herrmann, and E.J. Robertson. 1994. A primary requirement for nodal in the formation and maintenance of the primitive streak in the mouse. Development 120: 1919-1928.

Dale, L., G. Howes, B.M.J. Price, and J.C. Smith. 1992. Bone morphogenetic protein 4: A ventralizing factor in early $X e$ nopus development. Development 115: 573-585.

Dewulf, N., K. Verschueren, O. Lonnoy, A. Moren, S. Grimsby, K. Vande-Spiegle, K. Miyazono, D. Huylebroeck, and P. ten Dijke. 1995. Distinct spatial and temporal expression patterns of two type I receptors for bone morphogenetic proteins during mouse embryogenesis. Endocrinology 136: 26522663.

di Clemente, N., C. Wilson, E. Faure, L. Boussin, P. Carmillo, R. Tizard, J.-Y. Picard, B. Vigier, N. Josso, and R. Cate. 1994. Cloning, expression, and alternative splicing of the receptor for anti-Müllerian hormone. Mol. Endocrinol. 8: 1006-1020.

Ebner, R., R.-H. Chen, L. Shum, S. Lawler, T.F. Zioncheck, A. Lee, A.R. Lopez, and R. Derynck. 1993. Cloning of a type I TGF $\beta$ receptor and its effect on TGF $\beta$ binding to the type II receptor. Science 260: 1344-1348.

Estevez, M., L. Attisano, J.L. Wrana, P.S. Albert, J. Massagué, and D.L. Riddle. 1993. The Daf-4 gene encodes a bone morphogenetic protein receptor controlling C. elegans dauer larva development. Nature 365: 644-649.

Fainsod, A., H. Steinbeisser, and E.M. De Robertis. 1994. On the function of $B M P-4$ in patterning the marginal zone of the Xenopus embryo. EMBO 1. 13: 5015-5025.

Francis, P.H., M.K. Richardson, P.M. Brickell, and C. Tickle. 1994. Bone morphogenetic proteins and a signaling pathway that controls patterning in the developing limb bud. Development 120: 209-218.

Franzén, P., P. ten Dijke, H. Ichijo, H. Yamashita, P. Schulz, C.-H. Heldin, and K. Miyazono. 1993. Cloning of a TGF $\beta$ type I receptor that forms a heteromeric complex with the TGF beta type II receptor. Cell 75: 681-692.

Golden, J.W. and D.L. Riddle. 1984. A pheromone-induced developmental switch in Caenorhabditis elegans: Temperature-sensitive mutants reveal a wild-type temperature dependent process. Proc. Natl. Acad. Sci. 81: 819-823.

Graff, J.M., R.S. Thies, J.J. Song, A.J. Celeste, and D.A. Melton. 1994. Studies with a Xenopus BMP receptor suggest that ventral mesoderm-inducing signals override dorsal signals in vivo. Cell 79: 169-179.

Hayashi, Y., M. Koike, M. Matsutani, and T. Hoshino. 1988. Effects of fixation time and enzymatic digestion on immunohistochemical demonstration of bromodeoxyuridine in formalin-fixed, paraffin-embedded tissue. I. Histochem. Cytochem. 36: $511-514$.

Herrmann, B.G. 1991. Expression pattern of the Brachyury gene in whole-mount $T^{w^{\prime} i} / T^{w^{\prime} i}$ mutant embryos. Development 113: 913-917.

Holdener, B.C., C. Faust, N.S. Rosenthal, and T. Magnuson. 1994. $\mathrm{msd}$ is required for mesoderm induction in mice. Development 120: 1335-1346.

Johansson, B.M. and M.V. Wiles. 1995. Evidence for involvement of activin $A$ and bone morphogenetic protein 4 in mammalian mesoderm and haematopoietic development. Mol. Cell. Biol. 15: 141-151.

Jones, C.M., K.M. Lyons, and B.L.M. Hogan. 1991. Involvement of bone morphogenetic protein 4 (BMP-4) and $V g^{-1}-1$ in morphogenesis and neurogenesis in the mouse. Development 111: $531-542$.

Iones C.M., K.M. Lyons, P.M. Lapan, C.V.E. Wright, and B.L.M. Hogan. 1992. DVR-4 (bone morphogenetic protein-4) as a posterior-ventralizing factor in Xenopus mesoderm induction. Development 115: 639-647.

Kautman, M.H. 1990. In Postimplantation mammalian embryos: A practical approach. (ed. A.J. Copp and D.L. Cockroftl, pp. 81-91. IRL Press, Oxford, UK.

Kingsley, D.M. 1994a. The TGF- $\beta$ superfamily: New members, new receptors, and new genetic tests of function in different organisms. Genes \& Dev. 8: 133-146.

$1994 \mathrm{~b}$. What do BMPs do in mammals? Clues from the mouse short-ear mutation. Trends Genet. 10: 16-21.

Kingsley, D.M. , A.E. Bland, J.M. Grubber, P.C. Marker, L.B. Russell, N.G. Copeland, and N.A. Jenkins. 1992. The mouse short ear skeletal morphogenesis locus is associated with defects in a bone morphogenetic member of the TGF $\beta$ superfamily. Cell 71: 399-410.

Koenig B.B., J.S. Cook, D.H. Wolsing, J. Ting, J.P. Tiesman, P.E. Correa, C.A. Olson, A.L. Pecquet, F. Ventura, R.A. Grant, G.-X. Chen, J.L. Wrana, J. Massagué, and J.S. Rosenbaum. 1994. Characterization and cloning of a receptor for BMP-2 and BMP-4 from NIH 3T3 cells, Mol. Cell. Biol, 14: 59615974 .

Letsou, A., K. Arora, J.L. Wrana, K. Simin, V. Twombly, J. Jamal, K. Staehling-Hampton, F.M. Hoffmann, W.M. Gelbart, I Massagué, and M.B. O'Connor. 1995. Drosophila dpp signaling is mediated by the punt gene product: A dual ligandbinding type Il receptor of the TGF $\beta$ receptor family. Cell 80: 899-908.

Letterio, J.J., A.G. Geiser, A.B. Kulkarni, N.S. Roche, M.B. Sporn, and A.B. Roberts. 1994. Maternal rescue of transforming growth factor- $\beta 1$ null mice. Science 264: 1936-1938.

Lin, H.Y., X.-F. Wang, E. Ng-Eaton, R.A. Weinberg, and H.F Lodish. 1992. Expression cloning of the TGF $\beta$ type II receptor, a functional transmembrane serine/threonine kinase. Cell 68: 775-785.

Lyons, K.M., R.W. Pelton, and B.L.M. Hogan. 1989. Patterns of expression of murine Vgr-1 and BMP-2a RNA suggest that transforming growth factor- $\beta$-like genes coordinately regulate aspects of embryonic development. Genes \& Dev. 3: $1657-1668$.

Maéno, M., R.C. Ong, A. Suzuki, N. Ueno, and H.-F. Kung. 1994. A truncated bone morphogenetic protein 4 receptor alters the fate of ventral mesoderm to dorsal mesoderm: Roles of animal pole tissue in the development of ventral 
mesoderm. Proc. Natl. Acad. Sci. 91: 10260-10264.

Mansour, S.L., K.R. Thomas, and M.R. Capecchi. 1988. Disruption of the proto-oncogene int-2 in mouse embryo-derived stem cells: A general strategy for targeting mutations to nonselectable genes. Nature 336: 348-352.

Mathews L.S. and W.W. Vale. 1991. Expression cloning of an activin receptor, a predicted transmembrane serine kinase. Cell 65: 973-982.

Matzuk, M.M., T.R. Kumar, and A. Bradley. 1995. Different phenotypes for mice deficient in either activins or activin receptor type II. Nature 374: 356-360.

McMahon, A.P. and A. Bradley. 1990. The Wnt-1 (int-1) protooncogene is required for development of a large region of the mouse brain. Cell 62: 1073-1085.

Mishina, Y., A. Suzuki, D.J. Gilbert, N.G. Copeland, N.A. Jenkins, N. Ueno, and R.R. Behringer. 1995. Genomic organization and chromosomal location of the mouse type I BMP2/4 receptor. Biochem. Biophys. Res. Comm. 206: 310-317.

Nellen, D., M. Affolter, and K. Basler. 1994. Receptor serine/ threonine kinases implicated in the control of Drosophila body pattern by decapentaplegic. Cell 78: 225-237.

Okano, H., S. Yoshikawa, A. Suzuki, N. Ueno, M. Kaizu, M. Okabe, T. Takahashi, M. Matsumoto, K. Sawamoto, and K. Mikoshiba. 1994. Cloning of a Drosophila melanogaster homolog of the mouse type-I bone morphogenetic proteins-2/ -4 receptor: A potential decapentaplegic receptor. Gene 148: 203-209.

Padgett, R.W., J.M. Wozney, and W.M. Gelbart. 1993. Human BMP sequences can confer normal dorsal-ventral patterning in the Drosophila embryo. Proc. Natl. Acad. Sci. 90: 29052909.

Penton, A., Y. Chen, K. Staehling-Hampton, J.L. Wrana, L. Attisano, J. Szidonya, J.A. Cassill, J. Massagué, and F.M. Hoffmann. 1994. Identification of two bone morphogenetic protein type I receptors in Drosophila and evidence that Brk25D is a decapentaplegic receptor. Cell 78: 239-250.

Power, M.-A. and P.P.L. Tam. 1993. Onset of gastrulation, morphogenesis and somitogenesis in mouse embryos displaying compensatory growth. Anat. Embryol. 187: 493-504.

Ray R.P., K. Arora, C. Nusslein-Volhard, and W.M. Gelbart. 1991. The control of cell fate along the dorsal-ventral axis of the Drosophila embryo. Development 113: 35-54.

Reddi, A.H. 1994. Bone and cartilage differentiation. Curr. Opin. Genet. Dev. 4: 737-744.

Ruberte, E., T. Marty, D. Nellen, M. Affolter, and K. Basler. 1995. An absolute requirement for both the type II and type I receptors, punt and thick veins, for dpp signaling in vivo. Cell 80: 889-897.

Sampath, T.K. and A.H. Reddi. 1981. Dissociative extraction and reconstitution of extracellular matrix components involved in local bone differentiation. Proc. Natl. Acad. Sci. 78: 7599-7603.

Sasaki, H. and B.L.M. Hogan. 1993. Differential expression of multiple fork head related genes during gastrulation and axial pattern formation in the mouse embryo. Development 118: 47-59.

Shawlot, W. and R.R. Behringer. 1995. Requirement of Lim 1 in head-organizer function. Nature 374: 425-430.

Shull, M.M., I. Ormsby, A.B. Kier, S. Pawlowski, R.J. Diebold, M. Yin, R. Allen, C. Sidman, G. Proetzel, D. Calvin, N. Annunziata, and T. Doetschman. 1992. Targeted disruption of the mouse transforming growth factor- $\beta 1$ gene results in multifocal inflammatory disease. Nature 359: 693-699.

Snow, M.H.L. 1977. Gastrulation in the mouse: Growth and reorganization of the epiblast. I. Embryol. Exp. Morphol. 42: 293-303.
Soriano, P., C. Montgomery, R. Geske, and A. Bradley. 1991. Targeted disruption of the $c$-src proto-oncogene leads to osteopetrosis in mice. Cell 64: 693-702.

Suzuki, A., N. Shioda, T. Maeda, M. Tada, and N. Ueno. 1994a. A mouse TGF- $\beta$ type I receptor that requires type II receptor for ligand binding. Biochem. Biophys. Res. Comm. 298: 1063-1069.

Suzuki, A., R.S. Thies, N. Yamaii, J.J. Song, J.M. Wozney, K. Murakami, and N. Ueno. 1994b. A truncated bone morphogenetic protein receptor affects dorsal-ventral patterning in the Xenopus embryo. Proc. Natl. Acad. Sci. 91: 1025510259.

Tam, P.P.L. 1993. Histogenetic potency of embryonic tissues in ectopic sites. Methods Enzymol. 225: 164-190.

ten Diike, P., H. Ichijo, P. Franzén, P. Schulz, J. Saras, H. Toyoshima, C.-H. Heldin, and K. Miyazono. 1993. Activin receptor-like kinases: A novel subclass of cell-surface receptors with predicted serine/threonine kinase activity. Oncogene 8: 2879-2887.

ten Dilke, P., H. Yamashita, T.K. Sampath, A.H. Reddi, M. Estevez, D.L. Riddle, H. Ichijo, C.-H. Heldin, and K. Miyazono. 1994. Identification of type I receptors for osteogenic protein-1 and bone morphogenetic protein-4. 1. Biol. Chem. 269: 16985-16988.

Urist, M.R. 1965. Bone formation by autoinduction. Science 150: 893-899.

Winnier, G., M. Blessing, P.A. Labosky, and B.L.M. Hogan. 1995. Bone morphogenetic protein-4 (BMP-4) is required for mesoderm formation and patterning in the mouse. Genes \& Dev. 9: 2105-2116.

Wozney, I.M. 1992. The bone morphogenetic protein family and osteogenesis. Mol. Reprod. Dev, 32: 160-167.

Xie, T., A.L. Finelli, and R.W. Padgett. 1994. The Drosophila saxophone gene: A serine-threonine kinase receptor of the TGF- $\beta$ superfamily. Science 263: 1756-1759.

Yamaii, N., A.J. Celeste, R.S. Thies, J.J. Song, S.M. Bernier, D. Goltzman, K.M. Lyons, J. Nove, V. Rosen, and J.M. Wozney. 1994. A mammalian serine/threonine kinase receptor specifically binds BMP-2 and BMP-4. Biochem. Biophys. Res. Comm. 205: 1944-1951.

Zhao, G.-Q., X. Zhou, H. Eberspaecher, M. Solursh, and B. de Crombrugghe. 1993. Cartilage homeoprotein l, a homeoprotein selectively expressed in chondrocytes. Proc. Natl. Acad. Sci. 90: 8633-8637. 


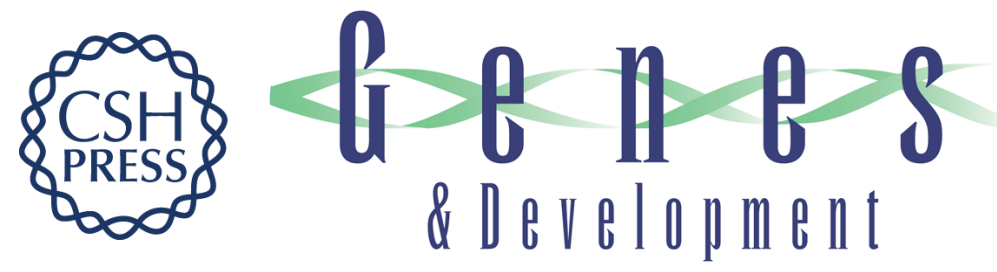

\section{Bmpr encodes a type I bone morphogenetic protein receptor that is essential for gastrulation during mouse embryogenesis.}

Y Mishina, A Suzuki, N Ueno, et al.

Genes Dev. 1995, 9:

Access the most recent version at doi:10.1101/gad.9.24.3027

References This article cites 64 articles, 27 of which can be accessed free at:

http://genesdev.cshlp.org/content/9/24/3027.full.html\#ref-list-1

License

Email Alerting

Service

Receive free email alerts when new articles cite this article - sign up in the box at the top right corner of the article or click here.

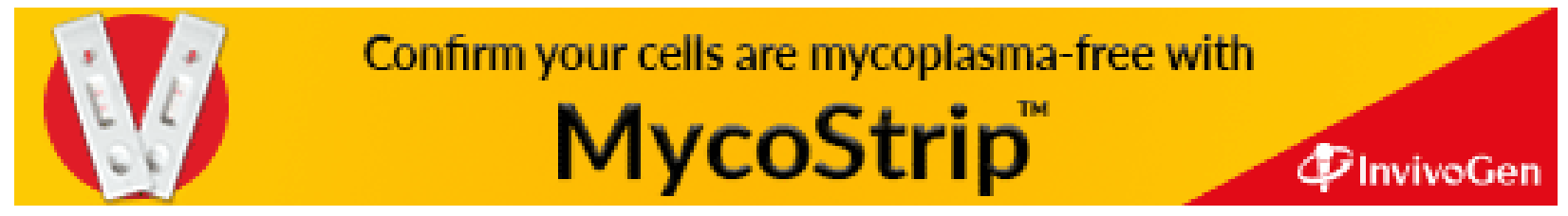

